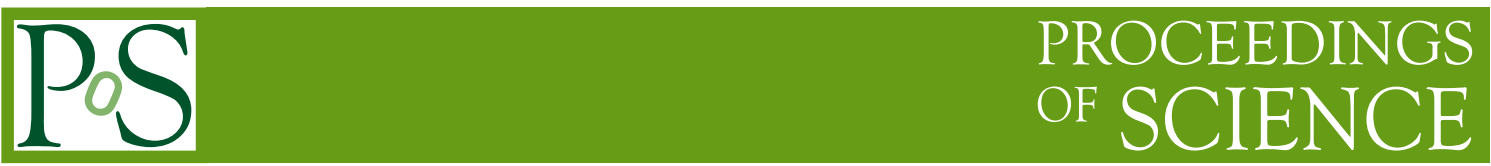

\title{
Wilson is not anomalous: on gauge anomalies in SMEFT
}

\author{
Quentin Bonnefoy, ${ }^{a}$ Luca Di Luzio, ${ }^{a, b, c}$ Christophe Grojean, ${ }^{a, d}$ Ayan Paul ${ }^{a, d}$ and \\ Alejo N. Rossia ${ }^{a, d, *}$ \\ ${ }^{a}$ DESY, \\ Notkestraße 85, 22607 Hamburg, Germany \\ ${ }^{b}$ Dipartimento di Fisica e Astronomia 'G. Galilei', Università di Padova, \\ Via F. Marzolo, 8 - 35131 Padova, Italy \\ ${ }^{c}$ INFN Sezione di Padova, \\ Via F. Marzolo, 8 - 35131 Padova, Italy \\ ${ }^{d}$ Institut für Physik, Humboldt-Universität zu Berlin, \\ Newtonstraße 15, 12489 Berlin, Germany \\ E-mail: quentin.bonnefoy@desy.de, luca.diluzio@unipd.it, \\ christophe.grojean@desy.de, ayan.paul@desy.de, alejo.rossia@desy.de
}

The use of Effective Field Theories (EFTs) in the search for NP is becoming more important given the lack of clear experimental signs of BSM physics. In particular, the Standard Model EFT (SMEFT) has become one of the most popular choices. Hence, the interest in revisiting EFTs is widespread in the community. On the other hand, the importance of chiral anomalies, either in gauge or global symmetries, cannot be overstated because of their phenomenological and formal importance. A natural question then arise: can higher-dimensional operators in an EFT generate gauge anomalies if the renormalizable part of the EFT is anomaly-free? We discuss whether dimension-6 operators in SMEFT can induce gauge anomalies. We find a negative answer and stress the importance of including all the relevant contributions in triangle-diagram computations. We provide arguments based on conserved currents and a more innovative derivation based on the construction of a bosonic EFT.

The Ninth Annual Conference on Large Hadron Collider Physics - LHCP2021

7-12 June 2021

Online

${ }^{*}$ Speaker 


\section{Introduction}

There is an increasing effort to study the formal aspects of Effective Field Theories. Anomalies in EFTs is a very rich topic, since it has both phenomenological and formal implications. The use of anomalies in axion and chiral Effective Field Theories (EFTs) exemplifies the former, while the impact of gauge anomalies on a gauge quantum field theory justifies the latter [1,2].

The Standard Model Effective Field Theory (SMEFT) is one of the most popular EFTs in collider searches for New Physics (NP). It is build upon the Standard Model and it shares its anomaly-free field content. Hence, it was naively expected that SMEFT would be free of gauge anomalies. However, the role of new high-dimensional chiral interactions in gauge anomalies was reassessed recently [3-5]. The set of dimension-6 operators [6],

$$
\begin{aligned}
& O_{\varphi \psi_{R}}=i \frac{c_{\varphi \psi, i j}}{\Lambda^{2}}\left(\varphi^{\dagger} \overleftrightarrow{D}_{\mu} \varphi\right) \bar{\psi}_{R}^{i} \gamma^{\mu} \psi_{R}^{j}, \\
& O_{\varphi \psi_{L}}^{(1)}=i \frac{c_{\varphi \psi, i j}^{(1)}}{\Lambda^{2}}\left(\varphi^{\dagger} \overleftrightarrow{D}_{\mu} \varphi\right) \bar{\psi}_{L}^{i} \gamma^{\mu} \psi_{L}^{j}, \quad O_{\varphi \psi_{L}}^{(3)}=i \frac{c_{\varphi \psi, i j}^{(3)}}{\Lambda^{2}}\left(\varphi^{\dagger} \overleftrightarrow{D}_{\mu}^{a} \varphi\right) \bar{\psi}_{L}^{i} \tau^{a} \gamma^{\mu} \psi_{L}^{j},
\end{aligned}
$$

are of special interest since they modify the fermion gauge vertices in a chiral fashion. Consequently, it seems possible that they might break the anomaly cancellation guaranteed at dimension 4 by the SM hypercharge assignment.

We revise this statement first in an abelian toy model by applying canonical techniques and show that such operators do not yield new anomaly cancellation conditions. Then, we arrive at the same conclusions by using bosonic EFT techniques. Finally, we apply the latter techniques to the more complex case of the SMEFT at dimension 6, focusing on its neutral EW sector.

\section{Current arguments in a toy model}

Let us a consider a toy model with two left-handed ( $\mathrm{LH})$ and two right-handed (RH) Weyl fermions, arranged in two Dirac fermions $\psi_{k=1,2}$, a complex scalar $\varphi$ and a gauge field $A_{\mu}$ of field strength $F_{A}$. Its Lagrangian density is,

$$
\mathcal{L}=-\frac{F_{A, \mu v}^{2}}{4 g_{A}^{2}}+|\partial \varphi|^{2}-V(|\varphi|)+i \bar{\psi}_{k} \not D \psi_{k}+i \sum_{H=L, R} \frac{c_{H, k}}{\Lambda^{2}}\left(\varphi^{\dagger} \overleftrightarrow{\partial}_{\mu} \varphi\right) \bar{\psi}_{H, k} \gamma^{\mu} \psi_{H, k},
$$

where $D_{\mu} \psi_{k}=\left(\partial_{\mu}+i q_{k}^{A} A_{\mu}\right) \psi_{k}$ contains a vector-like coupling to $A^{1}$. Note that $\varphi$ is uncharged under the gauge symmetry $U(1)_{A}$ of $A$ and we consider massless fermions. We do not include bare mass terms for the fermions since we want to study which chiral symmetries of Eq. (2) can be consistently gauged. We also postpone the inclusion of Yukawa couplings to Section 3.

This model has a chiral (more precisely axial) global symmetry under which $\varphi \rightarrow e^{i q_{\varphi}^{B} \epsilon_{B}} \varphi, \psi_{k} \rightarrow$ $e^{i q_{k}^{B} \gamma_{5} \epsilon_{B}} \psi_{k}$, and whose associated Noether current is

$$
J_{\mu}^{B}=-i q_{\varphi}^{B}\left(-\varphi^{\dagger} \overleftrightarrow{\partial}_{\mu} \varphi+2 i \sum_{H=L, R} \frac{c_{H, k}}{\Lambda^{2}}|\varphi|^{2} \bar{\psi}_{H, k} \gamma_{\mu} \psi_{H, k}\right)+q_{k}^{B} \bar{\psi}_{k} \gamma_{\mu} \gamma_{5} \psi_{k}
$$

\footnotetext{
${ }^{1}$ Summation over repeated indices is implicit.
} 
This current is conserved at classical level, i.e. $\partial^{\mu} J_{\mu}^{B}=0$. Since we would like to gauge it, we ask when it is anomaly free. The usual anomaly cancellation at dimension 4 would enforce,

$$
U(1)_{A}^{2} \times U(1)_{B}:\left(q_{k}^{A}\right)^{2} q_{k}^{B}=0, \quad U(1)_{B}^{3}:\left(q_{k}^{B}\right)^{3}=0,
$$

which implies $q_{1}^{B}=-q_{2}^{B}$ and, if $q_{k}^{B} \neq 0, q_{1}^{A}= \pm q_{2}^{A}$. The other anomalies vanish trivially.

Had we now gauged $U(1)_{B}$, the minimal Lagrangian would be

$$
\mathcal{L}=-\sum_{i=A, B} \frac{F_{i, \mu \nu}^{2}}{4 g_{i}^{2}}+|D \varphi|^{2}-V(|\varphi|)+i \bar{\psi}_{k} \not D \psi_{k}+i \sum_{H=L, R} \frac{c_{H, k}}{\Lambda^{2}}\left(\varphi^{\dagger} \overleftrightarrow{D}_{\mu} \varphi\right) \bar{\psi}_{H, k} \gamma^{\mu} \psi_{H, k},
$$

where now $D_{\mu} \psi_{k}=\left(\partial_{\mu}+i q_{k}^{A} A_{\mu}+i q_{k}^{B} \gamma_{5} B_{\mu}\right) \psi_{k}, D_{\mu} \varphi=\left(\partial_{\mu}+i q_{\varphi}^{B} B_{\mu}\right) \varphi$. From now on, we will work in the broken phase of the theory, where we parametrize $\varphi=\frac{v+h}{\sqrt{2}} e^{i \frac{\theta}{v}}$, with real $v$. The Goldstone boson $\theta$ transforms under $U(1)_{B}$ as $\delta \theta=v q_{\varphi}^{B} \epsilon_{B}$, and the radial mode $h$ is inert under all symmetries.

Now, the expression of the classically-conserved Noether current can be written as,

$$
J_{\mu}^{B}=q_{k}^{B} \bar{\psi}_{k} \gamma_{\mu} \gamma_{5} \psi_{k}+q_{\varphi}^{B}\left(-v \partial_{\mu} \theta+\sum_{H=L, R} \frac{c_{H, k} v^{2}}{\Lambda^{2}} \bar{\psi}_{H, k} \gamma_{\mu} \psi_{H, k}\right)+\text { terms involving } h .
$$

The $h$-dependent terms cannot contribute to anomalies at one loop, so we focus on the pieces that depend on the GB $\theta$ only [4].

Let us study the diagrams that enter the computation of $\partial^{\mu}\left\langle 0\left|J_{\mu}^{B}(x) J_{v}^{A}(y) J_{\rho}^{A}(z)\right| 0\right\rangle$. The first term in the current above generates the usual anomalies that can be cancelled by imposing Eq. (4). The following terms, proportional to $q_{\varphi}^{B}$, generate the diagrams depicted in Fig. 1, which include a GB and fermion propagators and are sensitive to $c_{L / R}$. In the diagram on the left, there appears the three-point coupling between the GB and the fermions obtained from Eq. (2),

$$
\mathcal{L} \supset-\sum_{H=L, R} \frac{v c_{H, k}}{\Lambda^{2}} \partial_{\mu} \theta \bar{\psi}_{H, k} \gamma^{\mu} \psi_{H, k}
$$

The two diagrams in Fig. 1 cancel after contracting with $-(p+q)_{\mu}$ and we do not obtain any

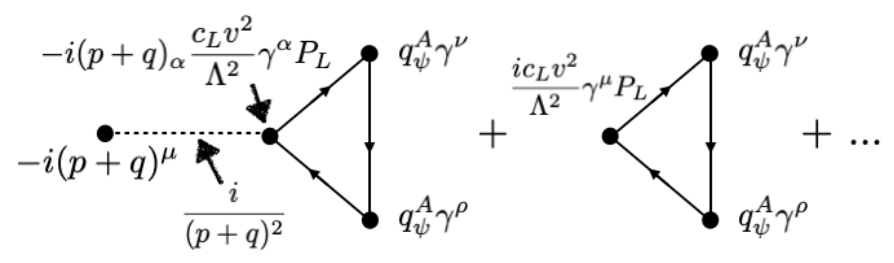

Figure 1: Triangle diagrams proportional to $q_{\varphi}^{B}$ (the $+\ldots$ refers to the same diagrams with the orientation of the fermionic arrows reversed). Solid lines are fermion propagators, the dashed one is a GB propagator and we indicate which combination of Dirac matrices and momenta enters each vertex.

constraint on $c_{L / R}$ from those triangles [4]. Instead, focusing only on the second diagram of Fig. 1, as proposed in Ref. [3] would yield the constraint $\left(q_{k}^{A}\right)^{2} q_{\varphi}^{B}\left(c_{L, k}-c_{R, k}\right)=0$, but this does not correspond to the full contribution of the Noether current. Consequently, $\partial^{\mu}\left\langle 0\left|J_{\mu}^{B}(x) J_{v}^{A}(y) J_{\rho}^{A}(z)\right| 0\right\rangle=0$ if and only if $\left(q_{k}^{A}\right)^{2} q_{k}^{B}=0$, and in particular we do not find any condition on $c_{L / R}$. The same result holds in the unbroken phase $[4,7,8]$. 


\section{Bosonic EFT techniques}

If we add to the toy model in Eq. (5) the Yukawa terms $\delta \mathcal{L}=-y_{k} \varphi \bar{\psi}_{k, L} \psi_{k, R}+$ h.c., with $y_{k}$ real, the charged chiral fermions acquire a mass in the broken phase. Gauge invariance forces that $2 q_{k}^{B}=q_{\varphi}^{B}$ and the Noether current in Eq. (3) respects the same anomalous Ward identities as in the massless-fermions case [9, 10]. Now, one can define a bosonic EFT below the fermion mass where the anomalies of the heavy fields are encoded in Wess-Zumino (WZ) terms [1, 11, 12]. We are going to show that no such WZ terms proportional to the dimension-6 WCs are generated.

The possible WZ terms for our toy model are,

$$
\mathcal{L}_{\mathrm{EFT}}=-\frac{C_{A A}}{16 \pi^{2}} \frac{\theta}{v} F_{A} \tilde{F}_{A}-\frac{C_{A B}}{16 \pi^{2}} \frac{\theta}{v} F_{A} \tilde{F}_{B}-\frac{C_{B B}}{16 \pi^{2}} \frac{\theta}{v} F_{B} \tilde{F}_{B}-\frac{E_{A B A}}{8 \pi^{2}} A_{\mu} B_{v} \tilde{F}_{A}^{\mu \nu}-\frac{E_{A B B}}{8 \pi^{2}} A_{\mu} B_{v} \tilde{F}_{B}^{\mu \nu} .
$$

The coefficients can be computed from fermion triangle diagrams (see details in App. B of Ref. [4]) and the result is

$$
C_{A A}=\left(q_{k}^{A}\right)^{2}, \quad C_{A B}=0, \quad C_{B B}=\frac{2\left(q_{k}^{B}\right)^{2}}{3}, \quad E_{A B A}=\frac{4\left(q_{k}^{A}\right)^{2} q_{k}^{B}}{3}, \quad E_{A B B}=0 .
$$

Hence, they vanish after imposing the usual anomaly cancellation conditions in Eq. (4) and there is no dependence on the dimension- 6 Wilson coefficients.

The same procedure can be applied to a modified version of the SMEFT in which we add a right-handed neutrino and modify the gauge and Yukawa coupling values such that it makes sense to integrate out all the fermions while keeping the gauge fields in the EFT. Despite these modifications, our results are valid for the most general case of SMEFT.

The Wess-Zumino terms to compute for the neutral electroweak sector of SMEFT are:

$$
\begin{aligned}
\mathcal{L}_{\mathrm{EFT}} \supset & -\frac{C_{F_{0} B}}{16 \pi^{2}} \frac{\pi^{3}}{v} F_{0} \tilde{B}-\frac{C_{F_{0} W^{3}}}{16 \pi^{2}} \frac{\pi^{3}}{v} F_{0} \tilde{W}^{3}-\frac{E_{B W^{3} F_{0}}}{8 \pi^{2} \Lambda^{2}} B_{\mu} W_{v}^{3} \tilde{F}_{0}^{\mu v}-\frac{E_{A_{0} W^{3} W^{3}}}{8 \pi^{2} \Lambda^{2}} A_{0, \mu} W_{v}^{3} \tilde{W}^{3, \mu \nu} \\
& -\frac{E_{A_{0} B B}}{8 \pi^{2} \Lambda^{2}} A_{0, \mu} B_{v} \tilde{B}^{\mu \nu}-\frac{E_{A_{0} W^{3} B}}{8 \pi^{2} \Lambda^{2}} A_{0, \mu} W_{v}^{3} \tilde{B}^{\mu \nu}-\frac{E_{A_{0} B W^{3}}}{8 \pi^{2} \Lambda^{2}} A_{0, \mu} B_{v} \tilde{W}^{3, \mu \nu},
\end{aligned}
$$

where we have defined $A_{0, \mu} \equiv v^{2}\left(y_{\varphi} B_{\mu}-\frac{W_{\mu}^{3}}{2}\right)-v \partial_{\mu} \pi^{3}$ and $\pi^{3}$ is the GB to be absorbed by the $Z$. The explicit computation of the Wilson coefficients shows that the conditions on the hypercharges needed to cancel the anomalies in the SM is enough to make these terms (hence their anomalous gauge variation) vanish and that the dimension-6 operators do not generate any further anomaly [4].

\section{Conclusions}

We showed how to compute anomalies in an EFT consistently and stressed the importance of including the Goldstone bosons in the computation. Rephrasing our discussion in terms of Wess-Zumino terms allowed us to extend our results to the SMEFT. In that case, we showed that dimension- 6 operators do not generate any new anomaly in the neutral sector of the SMEFT. 


\section{Acknowledgements}

We thank Fady Bishara, Emanuele Gendy, Di Liu, and Philipp Englert for useful discussions. This work is supported by the Deutsche Forschungsgemeinschaft under Germany's Excellence Strategy EXC 2121 "Quantum Universe" - 390833306. The work of C.G. and A.R. was also supported by the International Helmholtz-Weizmann Research School for Multimessenger Astronomy, largely funded through the Initiative and Networking Fund of the Helmholtz Association. The work of L.D.L. is supported by the Marie Skłodowska-Curie Individual Fellowship grant AXIONRUSH (GA 840791).

\section{References}

[1] J. Preskill, "Gauge anomalies in an effective field theory," Annals Phys. 210 (1991), 323-379 doi:10.1016/0003-4916(91)90046-B.

[2] A. Bilal, "Lectures on Anomalies," [arXiv:0802.0634 [hep-th]].

[3] O. Cata, W. Kilian and N. Kreher, "Gauge anomalies in the Standard-Model Effective Field Theory," [arXiv:2011.09976 [hep-ph]].

[4] Q. Bonnefoy, L. Di Luzio, C. Grojean, A. Paul and A. N. Rossia, "Comments on gauge anomalies at dimension-six in the Standard Model Effective Field Theory," JHEP 05 (2021), 153 doi:10.1007/JHEP05(2021)153 [arXiv:2012.07740 [hep-ph]].

[5] F. Feruglio, "A Note on Gauge Anomaly Cancellation in Effective Field Theories," JHEP 03 (2021), 128 doi:10.1007/JHEP03(2021)128 [arXiv:2012.13989 [hep-ph]].

[6] B. Grzadkowski, M. Iskrzynski, M. Misiak and J. Rosiek, "Dimension-Six Terms in the Standard Model Lagrangian,” JHEP 10 (2010), 085 doi:10.1007/JHEP10(2010)085 [arXiv: 1008.4884 [hep-ph]].

[7] S. L. Adler, "Axial vector vertex in spinor electrodynamics," Phys. Rev. 177 (1969), 2426-2438 doi:10.1103/PhysRev.177.2426

[8] J. S. Bell and R. Jackiw, "A PCAC puzzle: $\pi^{0} \rightarrow \gamma \gamma$ in the $\sigma$ model," Nuovo Cim. A 60 (1969), 47-61 doi:10.1007/BF02823296

[9] D. J. Gross and R. Jackiw, "Effect of anomalies on quasirenormalizable theories," Phys. Rev. D 6 (1972), 477-493 doi:10.1103/PhysRevD.6.477

[10] C. Bouchiat, J. Iliopoulos and P. Meyer, "An Anomaly Free Version of Weinberg's Model," Phys. Lett. B 38 (1972), 519-523 doi:10.1016/0370-2693(72)90532-1

[11] J. Wess and B. Zumino, "Consequences of anomalous Ward identities," Phys. Lett. B 37 (1971), 95-97 doi:10.1016/0370-2693(71)90582-X

[12] E. Witten, "Global Aspects of Current Algebra," Nucl. Phys. B 223 (1983), 422-432 doi:10.1016/0550-3213(83)90063-9 